\title{
Improvement of antibacterial efficacy using antibiotic encapsulated silica-containing redox nanoparticles
}

\author{
Han Ngoc Tran ${ }^{1,2}$, Nhu-Thuy Trinh ${ }^{2,3}$, Hoai-Ngan Thien Pham ${ }^{1,2}$, Hanh Thi Ngoc Nguyen ${ }^{4}$, Long Binh Vong ${ }^{2,3}$, $^{*}$
}

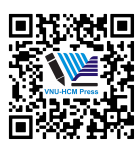

Use your smartphone to scan this QR code and download this article

${ }^{1}$ Faculty of Biology and Biotechnology, University of Science Ho Chi Minh city

${ }^{2}$ Vietnam National University Ho Chi Minh city

${ }^{3}$ School of Biomedical Engineering, International University Ho Chi Minh city

${ }^{4}$ School of Biotechnology, Hong Bang International University

\section{Correspondence}

Long Binh Vong, Vietnam National University Ho Chi Minh city

School of Biomedical Engineering, International University Ho Chi Minh city

Email: vblong@hcmiu.edu.vn

History

- Received: 2020-11-16

- Accepted: 2021-02-23

- Published: 2021-03-11

DOI : 10.32508/stdj.v24i1.2495

\section{Check for updates}

\section{Copyright}

(c) VNU-HCM Press. This is an openaccess article distributed under the terms of the Creative Commons Attribution 4.0 International license.

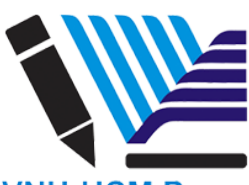

VNU-HCM Press

\begin{abstract}
Purpose: Improving solubility and antibacterial efficiency of cephalothin by using silica-containing redox nanoparticle (siRNP) as a system to encapsulate and deliver this hydrophobic antibiotic. Methods : siRNP was synthesized by assembling amphiphilic block copolymers possessing a reactive oxygen species scavenging nitroxide radical and drug absorptive silica moieties in a hydrophobic side chain. Cephalothin, a hydrophobic antibiotic, was encapsulated into siRNP (cephalothin@siRNP) by mixing and dialysis methods. Antibacterial activity of cephalothin@siRNP against Staphylococcus aureus (S. aureus) và Escherichia coli (E. coli) was evaluated by the agar diffusion method. Results: The average size of siRNP and cephalothin@siRNP was $43.83 \mathrm{~nm}$ and 50.15 $\mathrm{nm}$, respectively. After encapsulation in siRNP, the solubility of cephalothin was improved compared to cephalothin in an aqueous solution. The result showed that in vitro antibacterial activities of cephalothin and cephalothin@siRNP had no statistical difference after $24 \mathrm{~h}$ incubation on agar plates on both S. aureus and E. coli. However, after an extended incubation time, regrowth of $E$. coli colonies in the inhibitory zone was found in cephalothin treated plate. Interestingly, E. coli regrowth was significantly reduced in plates treated with cephalothin@siRNP. Conclusion: In this study, siRNP successfully encapsulated cephalothin and enhanced the solubility of this drug. The antibacterial activity of cephalothin is prolonged when encapsulated in siRNP, which suppressed the reccurrence of E. coli colonies. Cephalothin@siRNP has the potential to inhibit antibiotic resistance.
\end{abstract}

Key words: antibiotic resistance, infectious disease, redox nanoparticles, antimicrobial activity, ROS

\section{INTRODUCTION}

Nowadays, indiscriminate use of antibiotics has increased the uncontrollable development of many multidrug resistance (MDR) bacteria, becoming one of the most dangerous threats to public health. Infection of MDR into the community lead to increased morbidity, mortality, higher expenditures for health care, and grade of using antibiotics ${ }^{1}$. Although many types of antibiotics have been developed, demand for antibacterial infection treatment are still an urgent need as the number of resistance of bacterial strains is increasing ${ }^{2}$. One of the main bacterial resistance mechanisms has been reported to be associated with the overproduction of reactive oxygen species (ROS) ${ }^{3}$. Under microbial infection , not only inflammatory response but also the use of antibiotics induce ROS overproduction. ROS overproduction might induce gene mutation, which activated the bacterial antibiotic resistance mechanism ${ }^{4}$. On the other hand, several antibiotics such as cephalothin, erythromycin, rifamycins, gentamycin, kanamycin... present a low bioavailability due to low water solubility, low stability under physiological environments ${ }^{5}$. Therefore developing new efficacy antibacterial method to improve bioavailability is urgently necessary. In recent years, there has been a growing trend of enhancing the efficacy of available antibiotics by using nanomaterials to carry and deliver them to improve their activities and solubility $^{6}$. However, conventional nanoparticles have exhibited a low drug loading capacity without or with low ROS scavenging activity. Previously, we developed silica-contaning redox nanoparticle (siRNP) with ROS scavening from nitroxide radical and drug absorption silica moieties in the core of the nanoparticle ${ }^{7}$. siRNP with core-shell form micellar nanoparticle in aqueous media was synthesized from an amphiphilic block copolymer composed of hydrophilic polyethylene glycol (PEG) and hydrophobic poly(silanoaminomethylstyreneblock-poly(4-(2,2,6,6-tetramethylpiperidine-1-oxyl) aminomethylstyrene) (siPMNT) (Figure 1). A stable nitroxide radical is covalently bonded with a hydrophobic chain via an amine linkage to scavenge ROS. At the same time, silica moieties form cross-linking to stabilize the structure of the 


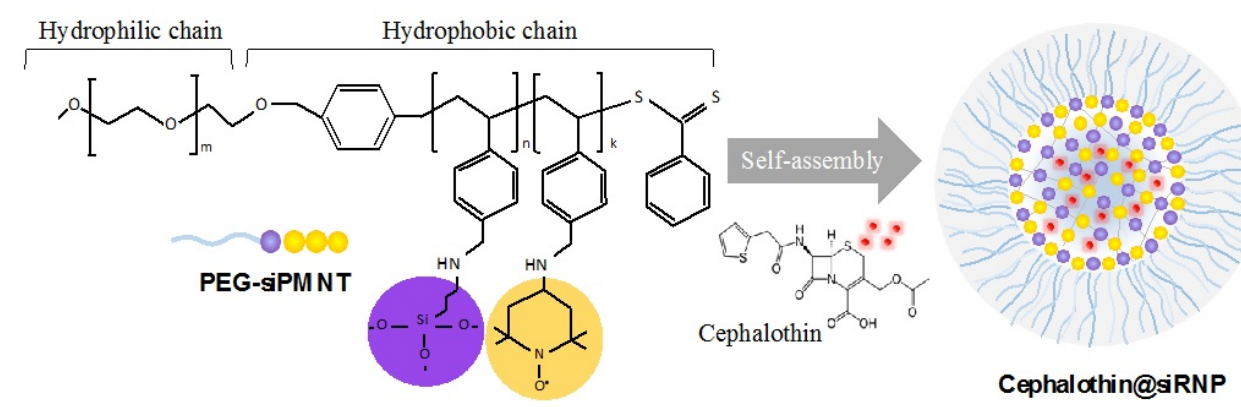

Figure 1: Structure of silica-contaning redox nanoparticle (siRNP) and cephalothin-loading siRNP (cephalothin@siRNP). siRNP was prepred by self-assembly of an amphiphilic block copolymer (PEG-siPMNT), which contains the ROS scavenging nitroxide radical moieties and drug absoption silica moieties. Cephalothin was encapsulated in the core of siRNP via the hydrophobic interaction and absorption on the silica.

nanoparticle and increase its loading drug capacity ${ }^{7}$. As an antibiotic model in this study, cephalothin, a generation of semi-synthetic antibiotic belonging to the cephalosporin group, kills and inhibits the bacterial growth by interrupting the synthesis of bacterial cells wall. However, low bioavailability of poorly water solubility and antibiotic resistance are the main challenges for using this antibiotic ${ }^{8}$. This study aims to evaluate the improvement of cephalothin solubility by loading cephalothin into siRNP (cephalothin@siRNP) and investigating the antibacterial activity.

\section{MATERIALS AND METHODS}

\section{Synthesis of siRNP and cephalothin@siRNP}

The silica-containing redox nanoparticle (siRNP) was prepared from amphiphilic PEG-siPMNT by dialysis, as described previously ${ }^{7}$. Briefly, $0.5 \mathrm{~mL}$ of PEG-siPMNT $(60 \mathrm{mg} / \mathrm{mL})$ was added to $0.5 \mathrm{~mL}$ of dimethylformamide (DMF, Wako Chemicals, Japan) and stirred well. The mixture was then transferred to a dialysis membrane (Spectra/Por, molecularweight cutoff size 3500; Spectrum Laboratories, CA) and dialyzed against water for $24 \mathrm{~h}$. Similarly, cephalothin@siRNP was prepared by dissolving cephalothin and PEG-siPMNT at a ratio of 1:10 in DMF and dialysis with the same protocol ${ }^{9}$.

\section{Mesurement of nanoparticle size}

Dynamic light scattering (DLS) measurement was used to evaluate particle sizes of siRNP and cephalothin@siRNP using Zetasizer Nano ZS (Malvern Instruments, Ltd., Malvern, UK) equipped with a $4-\mathrm{mW} \mathrm{He}-\mathrm{Ne}$ ion laser $(\lambda=633 \mathrm{~nm})$. The measurements were conducted at $25^{\circ} \mathrm{C}$ at a detection angle of $173^{\circ}$. siRNP was also observed by transmission electron microscope (TEM) imaging using a JEOL JEM-1400 instrument operated at $80 \mathrm{kV}$. The imaging samples were prepared by mounting a drop-cast onto a carbon-coated $\mathrm{Cu}$ grid and allowing it to dry in the air ${ }^{7}$.

\section{Evaluation of the DPPH free radical scav- enging capacity of siRNP}

The 2,2-diphenyl-1-picryl-hydrazyl-hydrate (DPPH, Tokyo Chemical Industry, Japan) free radical assay was conducted based on an electron-transfer that produces a violet solution in methanol. This free radical is reduced in the presence of an antioxidant molecule and leads to discoloration of the DPPH solution ${ }^{10}$. In this experiment, siRNP sample was diluted in water according to different concentrations with Tempo, and water served as the positive and negative control, respectively. A volume of $3 \mathrm{~mL}$ DPPH $0.1 \mathrm{mM}$ in methanol was added to $2 \mathrm{~mL}$ sample followed by incubation in the dark for 30 mins. Absorbances were measured at $517 \mathrm{~nm}$ by Spectro-UV11 UV-Vis spectrometers (MRC Laboratory Instruments, Ltd, Israel). The formula calculated DPPH scavenging efficacy:

$D P P H_{\text {scavenging efficacy }}=\frac{O D_{C}-O D_{S}}{O D_{C}} \times 100 \%$

Whereas:

ODs: the absorbance of the sample at $517 \mathrm{~nm}$ wavelength.

ODc: the absorbance of the negative control at 517 nm wavelength. 


\section{Evaluation of antibacterial activity}

The antibacterial activity of cephalothin and cephalothin@siRNP was determined by agar diffusion and minimum inhibitory concentration (MIC) assays. Briefly, a volume of $100 \mathrm{uL}$ of bacterial culture (Escherichia coli ATCC $^{\circledR} 25922^{\mathrm{TM}}$ or Staphylococcus aureus ATCC $^{\circledR} 23235^{\mathrm{TM}}$ ) on nutrient broth (NB) (containing $3 \mathrm{~g}$ meat extract, $5 \mathrm{~g}$ peptone, $5 \mathrm{~g} \mathrm{NaCl}$ ) and adjusted to 0.5 in MacFallen scale) was spread on a nutrient agar (NA) plate (containing $3 \mathrm{~g}$ meat extract, $5 \mathrm{~g}$ peptone, $5 \mathrm{~g} \mathrm{NaCl}, 10 \mathrm{~g}$ agar). An amount of $50 \mathrm{uL}$ of serial dilutions of cephalothin and cephalothin@siRNP was pumped to $6 \mathrm{~mm}$ wells on bacterial NA plates followed by incubation of $24 \mathrm{~h}$ at $37^{\circ} \mathrm{C}$. Namely, the cephalothin concentrations used to treat $S$. aureus were from 1 to $10 \mu \mathrm{g} / \mathrm{mL}$, while to treat E. coli were from 100 to $1000 \mu \mathrm{g} / \mathrm{mL}$. Sizes of inhibition zones and reoccurrence of bacterial colonies were recorded ${ }^{11}$. The MIC was also determined using the same concentrations of cephalothin or cephalothin@siRNP on the NB medium for $24 \mathrm{~h}$ treatment followed by an absorbance measurement at $620 \mathrm{~nm}$ using Spectro-UV11 UV-Vis spectrometers (MRC Laboratory Instruments, Ltd, Israel).

\section{Statistical analysis}

All values were expressed as mean \pm standard deviation (SD). The differences between the groups were examined for statistical significance using Student's $t$ test. A value of $\mathrm{p}<0.05$ was considered significant for all statistical analyses.

\section{RESULTS}

\section{Characterization of siRNP and cephalothin@siRNP}

We firstly evaluated the size and antioxidant activity of siRNP. The TEM image showed that siRNP presented a spherical shape (Figure 2A). By DLS measurements, the average size of siRNP was found at $43.83 \pm 1.95 \mathrm{~nm}$ and slightly increased to $50.15 \pm 0.78$ $\mathrm{nm}$ after drug encapsulation (Figure 2B). The antioxidant activity of siRNP was evaluated via DPPH scavenging capacity with different concentrations from 10 to $200 \mu \mathrm{g} / \mathrm{mL}$. As shown in Figure 2C, when siRNP concentration increased, the antioxidant activity of siRNP increased from $22.10 \%$ to $40.6 \%$, compared to tempo from $53.01 \%$ to $93.04 \%$. The results showed that siRNP had antioxidant activity and this activity was derived from the tempo radical attached in the core of nanoparticle.

We next evaluated the water solubility of cephalothin before and after encapsulation in nanoparticles. As shown in Figure 3, free cephalothin exhibited a partial solubility in water, which formed visible crystals precipitating in the bottom of the solution, suggesting the low bioavailability of these antibiotics for oral administration. When cephalothin was encapsulated into siRNP, the solution was clear and transparent, indicating that water solubility was significantly improved (Figure 3). These results suggested that siRNP can be utilized as a drug nanocarrier but also as an antioxidant agent, which may improve the therapeutic efficacy of the loaded antibiotics.

In Figure 4A, inhibition zones of cephalothin and cephalothin@siRNP against S. aureus and E. coli were determined by the agar diffusion method. The antibacterial activities of cephalothin and cephalothin@siRNP were plotted in Figure 4B, showing that cephalothin and cephalothin@siRNP performed antibacterial efficacy on both bacterial strains through large and clear inhibition zones on Petri plates. The diameter of inhibitory zone increased from 1.77 to $2.97 \mathrm{~cm}$ against $S$. aureus and from 1.63 to $2.00 \mathrm{~cm}$ against E. coli (Figure 4B). The results showed that cephalothin's antibacterial activity was more effective against Gram-positive bacteria ( $S$. aureus) than Gram-negative bacteria (E. coli). This is similar to previous studies from Hamilton-Miller et al. ${ }^{12}$ and Papich et al. ${ }^{13}$, which showed the antibacterial activity of cephalothin against Gram-positive bacteria was much higher than Gram-negative bacteria. However, statistical analysis on diameters of inhibition zones showed no statistically significant difference in antibacterial activities induced by cephalothin and cephalothin@siRNP (Figure 4B). To further investigate the antibacterial activity, we next evaluated the minimum inhibitory concentration (MIC) of cephalothin and cephalothin@siRNP against both bacteria. As shown in Figure 5A, there was no significant difference in the MIC of cephalothin and cephalothin@siRNP against $S$. aureus; however, we could observe a significant difference in the antibacterial activity of cephalothin@siRNP as compared to cephalothin in the E. coli strain (Figure 5B).

\section{Recurrence of $E$. coli in inhibition zones}

To further investigate the antibacterial activity of cephalothin@siRNP against E.coli, we further extended the incubation time on the agar diffusion assay to observe the colony's formation.

Regrowth of E. coli colonies was observed inside inhibitory zones of $E$. coli treated with cephalothin (Figure 6A). Interestingly, the number of regrowth 
A

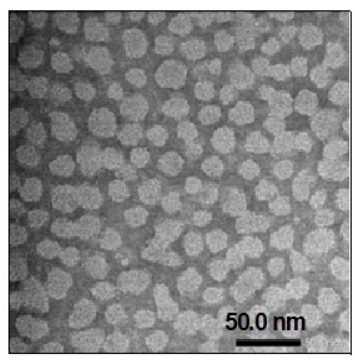

B

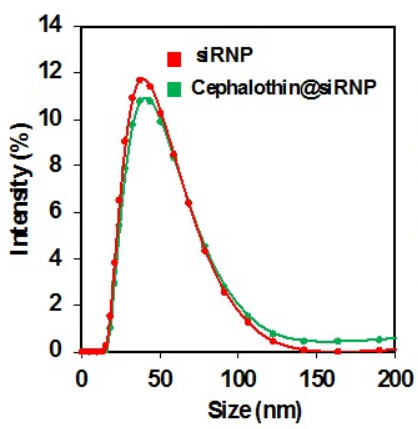

C

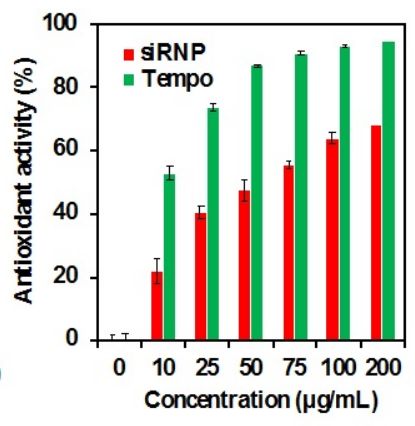

Figure 2: Characterization of siRNP. A) Transmission electron microscopy image of siRNP. B) Size distribution of siRNP and cephalothin@siRNP by dynamic light scattering (DLS) measurement. C) Antioxidant activity of siRNP by DPPH assay. Data express mean $\pm(S D), n=3$.

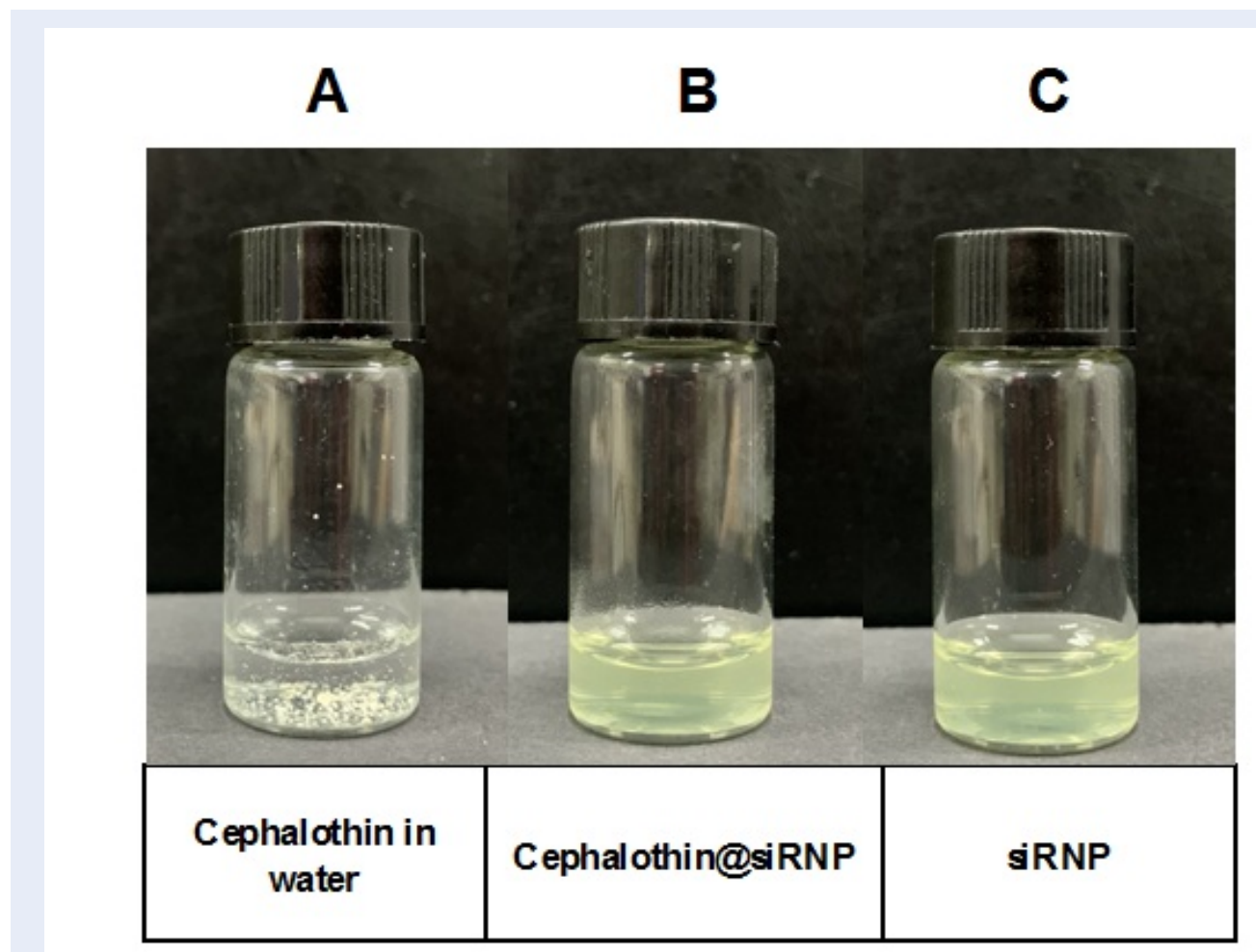

Figure 3: The solubility of cephalothin before and after encapsulation in siRNP. A) Solubility of cephalthin in water. B) cephalothin@siRNP. C) siRNP. Cephalothin in water exhibited low solubility with visible unsoluble particles, while cephalothin@siRNP was transparent in the aqueous solution. 
Antibacterial activity of cephalothin@siRNP

A
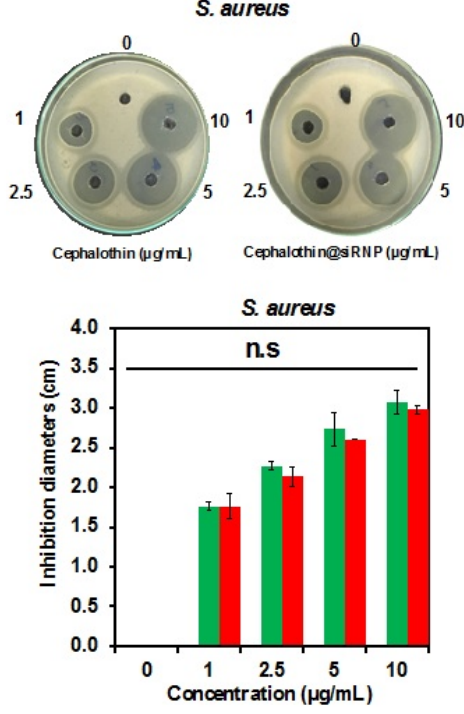

B

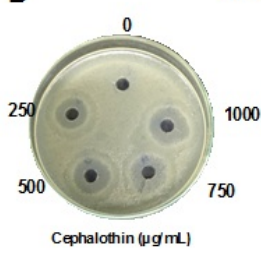

E. coli
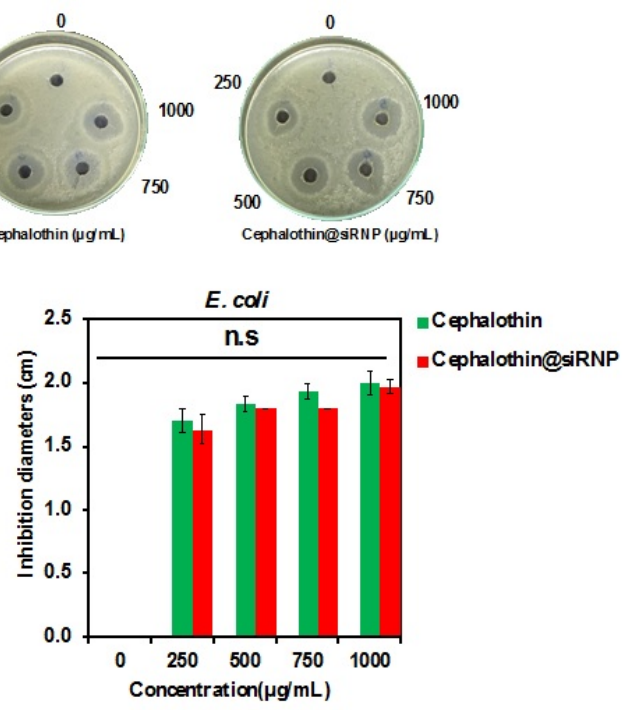

Figure 4: The antibaterial acitivity of cephalothin and cephalothin@siRNP. A) Inhibition zones of cephalothin and cephalothin@siRNP on S. aureus and E. coli on agar plates. B) Antibacterial activity of cephalothin and cephalothin@siRNP on S. aureus and E. coli. Data express mean $\pm(\mathrm{SD}), n=3$, n.s. indicates not significant $(p>$ 0.05). Concentrations shown in the figure indicated concentrations of cephalothin while concentrations of siRNP nanoparticles were 10 times higher than that of the drug.
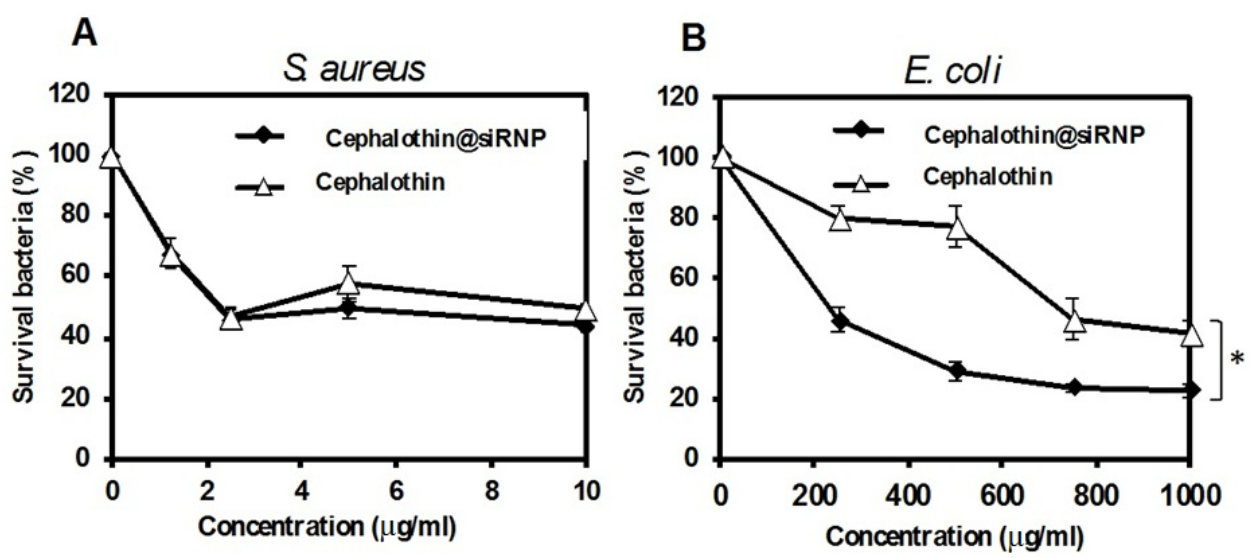

Figure 5: Minimum inhibitory concentration (MIC) of cephalothin and cephalothin@siRNP. (A) S. aureus. (B) E. coli. Data express mean $\pm(S D), n=3,{ }^{*} p<0.05$ 
A

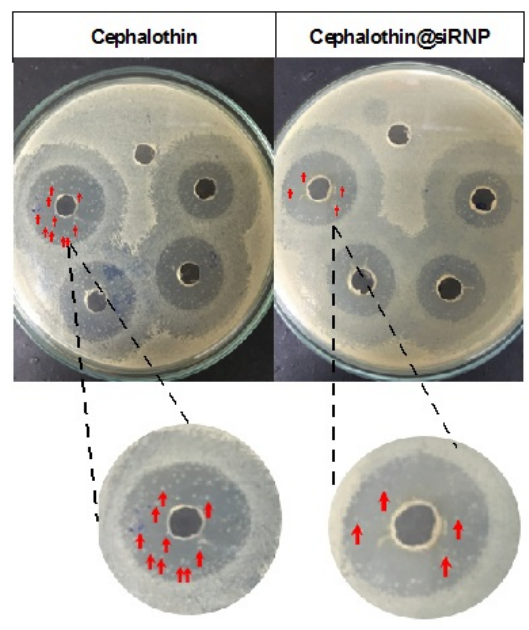

B

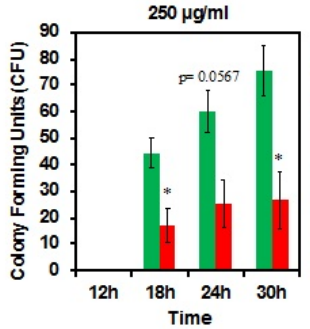

$750 \mu \mathrm{g} / \mathrm{ml}$

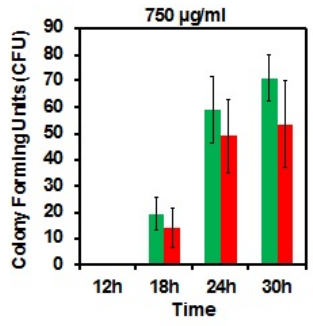

$500 \mu \mathrm{g} / \mathrm{ml}$
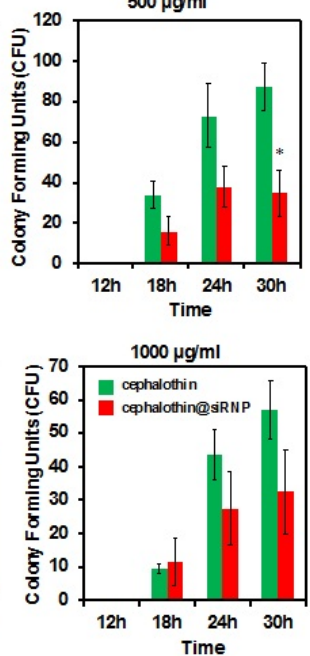

Figure 6: Recurrence of $E$. coli treated with cephalothin and cephalothin@siRNP after incubation with agar plate. A) The E. coli colony recurrence after $30 \mathrm{~h}$ incubation in agar plates (red arrows). B) Quantitative data of the $E$. coli colony recurrence after incubation periods on the agar plates. Data express mean $\pm(S D), n=3$ and * $p$ $<0.05$.

colonies was significantly reduced in plates treated with cephalothin@siRNP in comparison to plates treated with cephalothin. Moreover, at a 250 and $500 \mu \mathrm{g} / \mathrm{mL}$ concentration, the number of regrowing colonies of $E$. coli treated with free cephalothin was significantly lower than that treated with cephalothin@siRNP (Figure 6B). Specifically, at a concentration of $250 \mu \mathrm{g} / \mathrm{mL}$, after $30 \mathrm{~h}$ incubation, $76 \pm 7$ of the regrowth colonies were observed in the cephalothin-treated plates, while only $27 \pm 11$ colonies were found in cephalothin@siRNP-treated plates. Similarly at a $500 \mu \mathrm{g} / \mathrm{mL}$ concentration, quantilites of colonies of cephalothin@siRNP-treated sample was $35 \pm 11$, which was $39.77 \%$ reduction in comparision with the cephalothin-treated sample (Figure 6B). At higher concentrations (750 and $1000 \mu \mathrm{g} / \mathrm{mL}$ ), although the statistical difference between cephalothin and cephalothin@siRNP was not obtained, the lower number of CFU in cephalothin@siRNP was observed, indicating the siRNP contributed to the antibacterial feature of cephalothin to against the bacterial recurrence.

\section{DISCUSSION}

The current antibiotic drugs are insufficient therapy for the treatment of versatile infectious disease, and the development of new effective and safe drugs with high cost and time consuming are limited for applica- tions. Extention of antibiotic effectiveness for treatment of bacterial infection is required. Nanotechnology has been applied to overcome antibiotic resistance in various bacterial strains since nanoparticulate drug carriers exhibit excellent physiochemical features, enhanced drug uptake, specific targeting. Various nanoparticles have been developed to fight against antibacterial resistance, including liposomes, solid lipid nanoparticles, polymeric micelles, silver nanoparticles ${ }^{14-17}$. Current nanoparticles are merely designed as drug delivery systems and do not present the therapeutic effect. Low drug loading capacity and instability are other challenges of current nanocarriers ${ }^{18}$. In this study, siRNP was developed and presented high stability, and high drug loading capacity to encapsulate a hydrophobic antibiotic agent (cephalothin) and investigated the antibacterial property. In fact, we previously confirmed that siRNP with ROS scavenging capacity is a promising nanocarrier for the delivery of hydrophobic antiinflammatory and anti-cancer agents in the treatment of inflammation and cancer in mouse models ${ }^{7,19}$. Here, cephalothin@siRNP significantly improved solubility of cephalothin with the size about several tens of nanometer along with antioxidant feature via DLS measurement and DPPH assay (Fig. 2 and $\mathbf{3}$ ).

Cephalothin is known to be an $\beta$-lactam antibiotic that has strong coverage against Gram-positive 
strains, especially S.aureus, and moderate effect against some Gram-negative bacilli ${ }^{20}$. Regarding the antibacterial effect in this study, cephalothin encapsulated into siRNP exhibited antibacterial activity on both strains of $S$. aureus and E. coli. Although there was no difference when compared with cephalothin in the agar diffusion method, cephalothin@siRNP still showed a slightly stronger effect, especially in the MIC result on E. coli ( Fig. 5B). To some extent, using siRNP encapsulated cephalothin, which improved the antimicrobial effect of cephalothin against Gram-negative bacteria. These are the initial results for the research about nanomaterials' applications in improving the efficiency of previous generations of antibiotics, when here are the current research trends ${ }^{21,22}$.

Recurrence of E. coli in inhibition zones by agar diffusion test is an exciting investigation of this study. The results showed that cephalothin@siRNP was more effective at inhibiting the reinfection of E. coli than cephalothin (Fig. 6), which may be assumed that the encapsulation of cephalothin by siRNP can slowly release and enhanced the effect of cephalothin together with the ROS scavenging effect of siRNP. In a previous study, LoïcLéger et al. demonstrated that $\beta$-lactam antibiotics increased ROS production in both Gram-positive bacteria Enterococcus faecali and Gram-negative bacteria E. coli through the oxidation of membrane-associated demethylmenaquinone ${ }^{23}$. However, overproduction of ROS was reported to be one of the causes of antibiotic resistance in bacteria through gene mutation ${ }^{4}$. Hence, in this result, the ROS scavenging ability of nanoparticles maybe is a factor that prevents gene mutation of bacterial in drug resistance. These results demonstrated the potential of applying siRNP in treating antibiotic resistant bacteria.

\section{CONCLUSIONS}

In this study, cephalothin was successfully encapsulated by a silica-containing redox nanoparticle (siRNP) to form cephalothin@siRNP, which significantly improved the solubility of cephalothin. The antibacterial results showed that cephalothin@siRNP exhibited antibacterial activity against both Grampositive $S$. aureus strain and Gram-negative E. coli strain, but a significant improvement was obtained only in E. coli when comparing with the cephalothin-treated group. Interestingly, we found that cephalothin@siRNP significantly inhibited the recurrence of $E$. coli colonies after extending the incubation, suggesting the prolonged antibacterial activity of cephalothin@siRNP. Further investigations are being carried out to understand the underlying mechanism of this interesting phenomenon. Taken together, siRNP can improve the antibacterial effect and prevent MDR of low bioavailable antibiotics.

\section{LIST OF ABBREVIATIONS}

MDR multidrug resistance

siRNP silica-containing redox nanoparticle

PEG polyethylenglycol

siPMNT poly(silano aminomethylstyrene- blockpoly(4-(2,2,6,6-tetramethylpiperidine-1-oxyl)

aminomethylstyrene)

ROS reactive oxygen species

DLS dynamic light scattering

DPPH 2,2-diphenyl-1-picryl-hydrazyl-hydrate

DMF dimethylformamide

NB nutrient broth

NA nutrient agar

\section{COMPETING INTERESTS}

The authors have no competing financial interests to declare.

\section{AUTHORS' CONTRIBUTIONS}

Tran N H, Pham H T have contributed in conducting experiments, collection of data and interpretation of data. Tran N H, Nguyen H T N have prepared and revised manuscript. Vong B L, Trinh $\mathrm{N}$ have supervised, prepared and gave final approval of the manuscript to be submitted.

\section{ACKNOWLEDGEMENT}

This work was supported by Vietnam National Foundation for Science and Technology Development (NAFOSTED under Grant number 108.052017.327).

\section{REFERENCES}

1. Roberts RR, Hota B, Ahmad I, Scott RD, 2nd, Foster SD, Abbasi $F$, et al. Hospital and societal costs of antimicrobialresistant infections in a Chicago teaching hospital: implications for antibiotic stewardship. Clinical Infectious Diseases. 2009;49(8):1175-1184. Available from: https://doi.org/10. 1086/605630PMid:19739972.

2. Tanwar J, Das S, Fatima Z, Hameed S. Multidrug resistance: an emerging crisis. Interdisciplinary Perspectives on Infectious Diseases. 2014;2014:541340. PMID: 25140175. Available from: https://doi.org/10.1155/2014/541340.

3. Mao W, Warren MS, Lee A, Mistry A, Lomovskaya O. MexXYOprM efflux pump is required for antagonism of aminoglycosides by divalent cations inPseudomonas aeruginosa. Antimicrobial Agents and Chemotherapy. 2001;45(7):20012007. PMID: 11408215. Available from: https://doi.org/10. 1128/AAC.45.7.2001-2007.2001.

4. Dwyer DJ, Kohanski MA, Collins JJ. Role of reactive oxygen species in antibiotic action and resistance. Current Opinion in Microbiology. 2009;12(5):482-489. PMID: 19647477. Available from: https://doi.org/10.1016/j.mib.2009.06.018. 
5. Sun T, Zhang YS, Pang B, Hyun DC, Yang M, Xia Y. Engineered nanoparticles for drug delivery in cancer therapy. Angewandte Chemie International Edition. 2014;53(46):1232012364. PMID: 25294565. Available from: https://doi.org/10. 1002/anie.201403036

6. Jiang L, Su C, Wen Y, Zhu Z, Liu J, He S, et al. Antibacterial activity and long-term stable antibacterial performance of nisin grafted magnetic GO nanohybrids. Materials Science and Engineering: C. 2020;111:110809. PMID: 32279808. Available from: https://doi.org/10.1016/j.msec.2020.110809.

7. Vong LB, Kimura S, Nagasaki Y. Newly Designed SilicaContaining Redox Nanoparticles for Oral Delivery of Novel TOP2 Catalytic Inhibitor for Treating Colon Cancer. Advanced Healthcare Materials. 2017;6(20):1700428. PMID: 28736844. Available from: https://doi.org/10.1002/adhm.201700428.

8. Jubeh B, Breijyeh Z, Karaman R. Antibacterial Prodrugs to Overcome Bacterial Resistance. Molecules. 2020;25(7):1543. PMID: 32231026. Available from: https://doi.org/10.3390/ molecules25071543.

9. Hossain MA, Yamashita M, Vong LB, Ikeda Y, Nagasaki Y. Silicainstalled redox nanoparticles for novel oral nanotherapeuticsimprovement in intestinal delivery with anti-inflammatory effects. Journal of Drug Targeting. 2014;22(7):638-647. PMID: 24955616. Available from: https://doi.org/10.3109/1061186X. 2014.928716.

10. Tsai CE, Lin LH. DPPH scavenging capacity of extracts from Camellia seed dregs using polyol compounds as solvents. Heliyon. 2019;5(8):e02315. PMID: 31485521. Available from: https://doi.org/10.1016/j.heliyon.2019.e02315.

11. Balouiri M, Sadiki M, Ibnsouda SK. Methods for in vitro evaluating antimicrobial activity: A review. Journal of Pharmaceutical Analysis. 2016;6(2):71-79. PMID: 29403965. Available from: https://doi.org/10.1016/j.jpha.2015.11.005.

12. Hamilton-Miller J, Kerry D, Brumfitt W. An in vitro comparison of cefoxitin, a semi-synthetic cephamycin, with cephalothin. The Journal of Antibiotics. 1974;27(1):42-48. PMID: 4843049. Available from: https://doi.org/10.7164/antibiotics.27.42.

13. Papich MG, Lindeman C. Cephalexin susceptibility breakpoint for veterinary isolates: Clinical Laboratory Standards Institute revision. Journal of Veterinary Diagnostic Investigation. 2018;30(1):113-120. PMID: 29145786. Available from: https: //doi.org/10.1177/1040638717742434.

14. Eleraky NE, Allam A, Hassan SB, Omar MM. Nanomedicine fight against antibacterial resistance: an overview of the recent pharmaceutical innovations. Pharmaceutics. 2020;12(2):142. PMID: 32046289. Available from: https://doi.org/10.3390/ pharmaceutics12020142.
15. Sharma M, Gupta N, Gupta S. Implications of designing clarithromycin loaded solid lipid nanoparticles on their pharmacokinetics, antibacterial activity and safety. RSC advances. 2016;6(80):76621-76631. Available from: https://doi.org/10. 1039/C6RA12841F.

16. Hill LE, Gomes CL. Characterization of temperature and $\mathrm{pH}$-responsive poly- $\mathrm{N}$-isopropylacrylamide-co-polymer nanoparticles for the release of antimicrobials. Materials Research Express. 2014;1(3):035405. Available from: https://doi.org/10.1088/2053-1591/1/3/035405.

17. Mukherjee S, Chowdhury D, Kotcherlakota R, Patra S. Potential theranostics application of bio-synthesized silver nanoparticles (4-in-1 system). Theranostics. 2014;4(3):316. PMID: 24505239. Available from: https://doi.org/10.7150/thno.7819.

18. Xu W, Ling P, Zhang T. Polymeric micelles, a promising drug delivery system to enhance bioavailability of poorly watersoluble drugs. Journal of drug delivery. 2013;2013. PMID: 23936656. Available from: https://doi.org/10.1155/2013/ 340315.

19. Nguyen THT, Trinh NT, Tran HN, Tran HT, Le PQ, Ngo DN, et al. Improving Silymarin oral bioavailability using silica-installed redox nanoparticle to suppress inflammatory bowel disease. Journal of Controlled Release. 2020;331:515-524. PMID: 33616078. Available from: https://doi.org/10.1016/j.jconrel. 2020.10.042.

20. Chaudhry SB, Veve MP, Wagner JL. Cephalosporins: A focus on side chains and $\beta$-lactam cross-reactivity. Pharmacy. 2019;7(3):103. PMID: 31362351. Available from: https://doi. org/10.3390/pharmacy7030103.

21. Dar MA, Ingle A, Rai M. Enhanced antimicrobial activity of silver nanoparticles synthesized by Cryphonectria sp. evaluated singly and in combination with antibiotics. Nanomedicine: Nanotechnology, Biology and Medicine. 2013;9(1):105-110. PMID: 22633901. Available from: https://doi.org/10.1016/j. nano.2012.04.007.

22. Vazquez-Muñoz R, Meza-Villezcas $A$, Fournier $P$, Soria-Castro E, Juarez-Moreno K, Gallego-Hernández A, et al. Enhancement of antibiotics antimicrobial activity due to the silver nanoparticles impact on the cell membrane. PloS One. 2019;14(11):e0224904. PMID: 31703098. Available from: https://doi.org/10.1371/journal.pone.0224904.

23. Léger L, Budin-Verneuil A, Cacaci M, Benachour A, Hartke A, Verneuil N. $\beta$-Lactam Exposure Triggers Reactive Oxygen Species Formation in Enterococcus faecalis via the Respiratory Chain Component DMK. Cell Reports. 2019;29(8):21842191. PMID: 31747593. Available from: https://doi.org/10. 1016/j.celrep.2019.10.080. 